38. VARIEDADES ENANAS DEL COMPLEJO DE HELICTOCHLOA ALBINERVIS-H. MARGINATA (POACEAE)

Carlos ROMERO-ZARCO

Recibido el 28 de octubre de 2015, aceptado para su publicación el 15 de noviembre de 2015

Dwarf varieties of the Helictochloa albinervis-H. marginata complex (Poaceae)

Palabras clave. Helictochloa, tipificación, gramíneas, Poaceae, Rif

Key words. Helictochloa, typification, grasses, Poaceae, Rif

El género Helictochloa Romero Zarco, está representado en el occidente de la región mediterránea por tres grupos de especies que han sido reconocidos con categoría de sección (Romero-Zarco, 2013). Las especies de la sección Scleravenastrum (Holub) Romero Zarco son de carácter acidófilo y distribución principalmente ibero-atlántica y magrebí, desde Francia hasta el Rif, pasando por la región Macaronésica. Incluye dos especies muy bien caracterizadas morfológicamente y con adaptaciones opuestas: H. levis (Hackel) Romero Zarco, planta cespitosa de hojas anchas, endémica de los esquistos de Sierra Nevada (1800-3000 m.s.m.), y H. hackelii (Henriq.) Romero Zarco, planta estolonífera de hojas setáceas, que sobrevive a duras penas entre las urbanizaciones costeras del SW de Portugal. El resto de la sección comprende un complejo poliploide $(2 n=14,28,42)$ cuyas especies más conocidas son $H$. albinervis (Boiss.) Romero Zarco y H. marginata (Lowe) Romero Zarco [anteriormente conocida como Avenula sulcata (Gay ex Boiss.) Dumort.]. La tercera especie de este complejo es $H$. cintrana
(Röser) Romero Zarco (Avenula occidentalis, nom. inval.), endémica del centro-oeste y SO de la Península.

En el año 1848, Reuter recolectó en el Sistema Central una forma reducida de H. marginata que etiquetó como "Avena carpetana", planta grácil con hojas más cortas y estrechas que el tipo de la especie, y con panículas menos desarrolladas. Plantas pirenaicas idénticas a la carpetana fueron descritas en 1977 como Avenula sulcata subsp. pyrenaica Holub (Holub, 1977). Un año más tarde, se describe una nueva forma endeble de esta especie pero, en este caso, creciendo en terrenos peridotíticos de la Sierra de Nogueira, en el NE de Portugal: Avenula delicatula Franco (Franco, 1978). Estas formas endebles fueron descritas con categoría varietal por Romero Zarco (1984) con el nombre de Avenula sulcata var. reuteri, en honor a su primer recolector conocido, una variante cuya presencia se ha observado en la mayor parte de los sistemas montañosos dentro del área de la especie, ocupando principalmente los lugares menos favorables para el buen desarrollo de los pastos. De igual manera, en Marruecos, en

Trabajo realizado en el marco del proyecto Flora Iberica IX(2) del Plan Nacional de 2012 del Ministerio de Economía y Competitividad (CGL2012-32914, cofinanciado por FEDER) y por el Plan Andaluz de Investigación de la Junta de Andalucía (RNM 204). Agradecemos al Conservatoire et Jardín Botaniques de la Ville de Genéve $(G)$ el envío de las fotografías del material citado por Saint-Yves y el permiso para su publicación. 


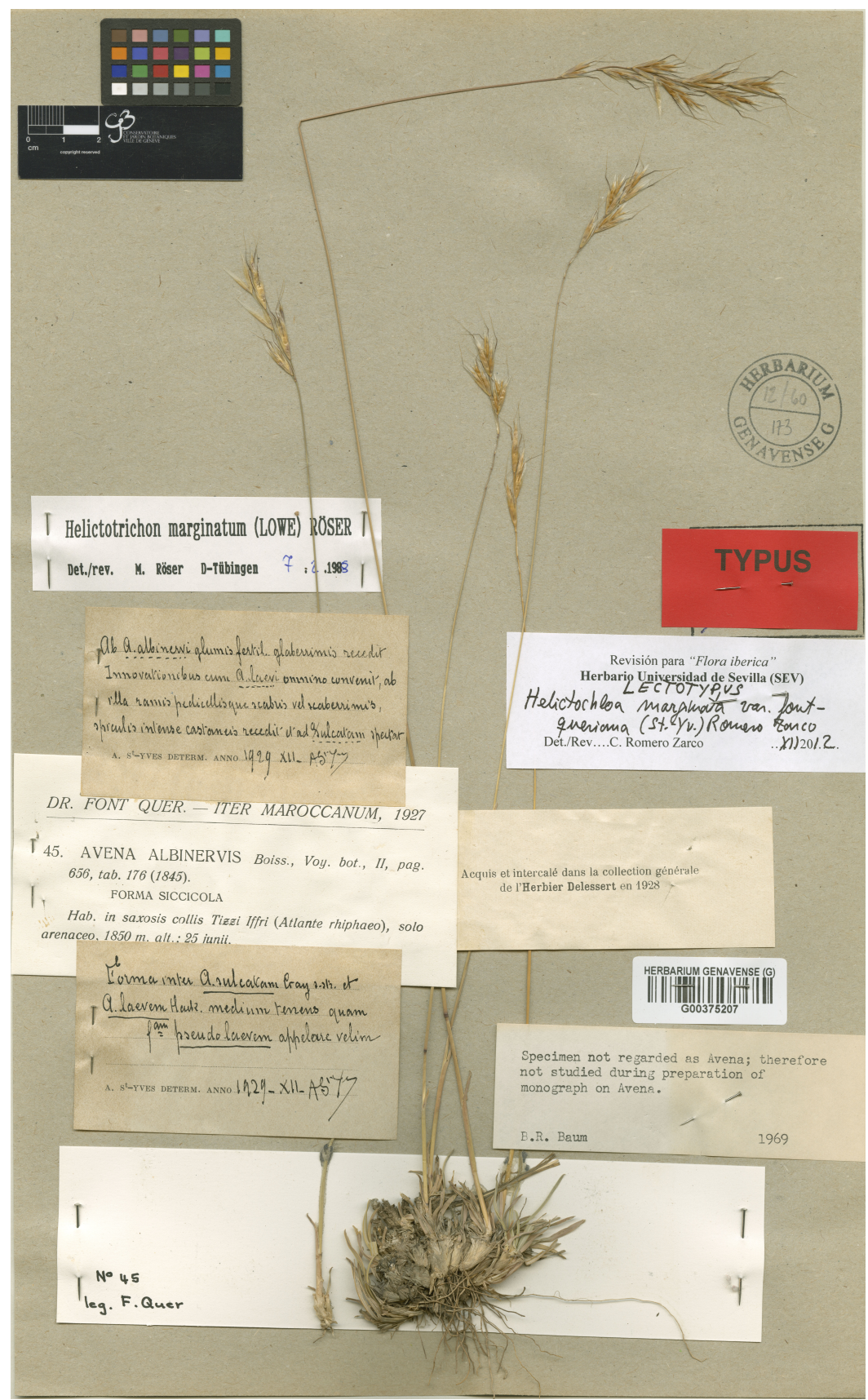

Figura 1. Lectotipo de Avena pratensis var. font-queriana St.-Yves. Fotografía publicada con permiso del Conservatorio y Jardín Botánico de Ginebra (Suiza). Lectotypus of Avena pratensis var. font-queriana St.-Yves. Photo published with permission of the Conservatoire et Jardin Botaniques de la Ville de Genéve (Switzerland). 
un paso o cerro llamado Tizi Ifri (Rif central), a 1850 m.s.m., Font Quer recolectó otra forma depauperada del mismo complejo, que distribuyó en su exsiccata Iter Maroccanum 1927 no. 45 como "Avena albinervis f. siccicola”. Un ejemplar de esta exsiccata del herbario Delessert (G 375207) fue descrito por Saint-Yves (1931) como Avena pratensis subsp. sulcata var. font-queriana St.-Yves.

En el contexto taxonómico del s. XX, Avena sulcata y Avena albinervis se separaban por la pelosidad del lema, que es glabro en la primera especie y tomentoso-seríceo en la parte inferior del dorso en la segunda. Tras los estudios de Röser (1989), se puso de manifiesto que el carácter no es del todo fiable, pues en algunas poblaciones de lo que hoy denominamos Helictochloa albinervis se mezclan plantas de lemas glabros con otras de lemas tomentososeríceos (Röser, 1989; 2006; Romero-Zarco, 2014). En los casos dudosos es necesario observar detalladamente la forma y longitud de las lígulas en las hojas basales. En $H$. marginata las lígulas basales miden $(1,5) 2-6 \mathrm{~mm}$ y son triangulares o triangular-acuminadas, mientras que en $H$. albinervis miden 0,5-1,5(3) mm y son truncadas o truncado-apiculadas.

La forma reducida del Rif ha sido confundida con el endemismo nevadense Helictochloa levis por algunos autores. El mismo Saint-Yves escribió en su etiqueta de revisión: "Forma inter A. salcatam Gay s.str. et A. laevem Hack. medium tenens quam f.am pseudolaevem appelare velim...". Pau (1932), en un breve comentario al final de un artículo sobre plantas rifeñas, afirmó "Y ahora recuerdo la Avena laevis [sic] Hackel herborizada por el Sr. FONT en el Atlas rifeño, y que la encuentro idéntica a la de Sierra Nevada de mi colección". Por su parte, Sennen \& Mauricio (1934: 129) señalaron también la presencia de Helictochloa levis (sub Avena) en el pico Tidiguine. En su monografía sobre Avenula, Romero-Zarco (1984) acepta arróneamente ambas citas para Marruecos, debido a que la planta de Font Quer estudiada era un ejemplar poco desarrollado y algo deteriorado. Röser (1989) advirtió el error y citó los ejemplares de la exsiccata de Font Quer conservados en los herbarios BC, BCF, G y MA en la lista de Helictochloa marginata (sub Helictotrichon). Más tarde, Romero-Zarco (1996) restituyó el estatus del endemismo nevadense, excluyendo su presencia en el NW de África (Rif), por lo que debe descartarse - por errónea- la cita para Melilla recogida en Anthos (Aedo, 2015) basada en las indicaciones de Pau (loc. Cit.).

Aclarado el asunto de la confusión de la planta rifeña con la nevadense, quedaba por explicar el hecho de que Font Quer la clasificara en Avena albinervis y, sin embargo, Saint-Yves la incluyera en Avena pratensis subsp. sulcata. En el pliego BC 68274 se mezclan plantas de lemas glabros con otras idénticas de lemas seríceos en la parte inferior. La etiqueta de revisión de Röser, fechada en 1988, es de "Helictotrichon albinerve", a pesar de que en su monografía no la recoge como tal. Se trata, por tanto, de una población heterogénea en lo que respecta al carácter de la pelosidad del lema. Este hecho, unido al examen de las lígulas, lleva a la conclusión de que dichas plantas se identifican mejor como una variedad de escaso desarrollo de Helictochloa albinervis que como una variedad de $H$. marginata.

En consecuencia, la nomenclatura de las plantas endebles del complejo quedaría como sigue:

Helictochloa marginata var. reuteri (Romero Zarco) Romero Zarco, comb. nov.

Basiónimo: Avenula sulcata var. reuteri Romero Zarco in Lagascalia 14: 122 (1984)

三 Avenula marginata var. reuteri (Romero Zarco) Romero Zarco in Acta Bot. Malacit. 18: 149 (1993)

三Avenula sulcata subsp. reuteri (Romero Zarco)

Franco in Silva Lusit. 5: 141 (1997).

$=$ Avenula delicatula Franco in Bot. J. Linn. Soc. 76(4): 359 (1978)

$=$ Avenula marginata subsp. pyrenaica Holub in 
Preslia 49(3): 208 (1977)

= Avenula lodunensis subsp. pyrenaica (Holub) Kerguélen

Tipo: "Sierra de Guadarrama ad viam Navacerrada... 16 Jul. 1848” (scripsit Reuter, G, holotypus).

Helictochloa albinervis var. font-queriana (St.-Yves) Romero Zarco, comb. nov.

Basiónimo: Avena pratensis var. font-queriana

St.-Yves in Candollea 4: 465 (1931)

$\equiv$ Helictochloa marginata var font-queriana

(St.-Yves) Romero Zarco in Candollea 66(1): 102 (2011).

Indicación locotípica: "Maroc espagnol: Tizzi Iffri, 1850 m., Font Quer Iter maroc. No 45 (herb. Deless.)".

Lectotipo (designado aquí): "Hab. in saxosis collis Tizzi Iffri (Atlanthe rhiphaeo), solo arenaceo, 1850 m. alt.; 25 junii”. Font Quer Iter maroc. 1929 no. 145 sub Avena albinervis f. siccicola (G 375207 , ejemplar asociado a las etiquetas de revisión de Saint-Yves). Fig.1.

En el mismo pliego que contiene al lectotipo elegido hay otro ejemplar de la misma exsiccata (isolectotipo, G 375207 a), sin etiqueta y montado en hoja aparte, que pertenece a la misma variedad y presenta los mismos caracteres descritos por Saint-Yves.

En los ejemplares de herbario revisados para Flora iberica antes de septiembre de 2015, se ha utilizado el epíteto font-queriana para las plantas que a partir de ahora han de llamarse $H$. marginata var. reuteri. Hasta la fecha no se han encontrado en la Península plantas semejantes a las rifeñas.

\section{Material estudiado.}

MARRUECOS. In saxosis collis Tizzi Iffri (Atlanthe rhiphaeo), solo arenaceo, $1850 \mathrm{~m}$. alt.; 25 junii 1850 Font Quer Iter Maroccanum, 1927 n $^{\circ}$ 45 Avena albinervis forma siccicola (BC 68274; G 375207; MA 8552).

\section{BIBLIOGRAFÍA}

AEDO, C. -2015- Anthos. Sistema de Información sobre las plantas de España. Real Jardín Botánico de Madrid. [http://anthos.es]. Fecha de consulta
22-X-2015.

FRANCO, J.A. -1978- Avenula (Dumort.) Dumort. En: V.H. Heywood (ed.): Flora Europaea Notulae Systematicae ad Floram Europaeam spectantes. No. 20. Short Notes (281). Bot. J. Linn. Soc. 76: 359-360.

HOLUB, J. -1977- Notes on some species of Avenula and Helictotrichon. Preslia 49(3): 203-321.

PAU, C. -1932- Plantas rifeñas. Cavanillesia 5: 175-177.

ROMERO-ZARCO, C. -1984- Revisión taxonómica del género Avenula (Dumort.) Dumort. (Gramineae) en la Península Ibérica e Islas Baleares. Lagascalia 14: 39-146.

ROMERO-ZARCO, C. -1996- Contribución al conocimiento de las gramíneas endémicas de la Península Ibérica. Anales Jardín Bot. Madrid 54(2): 528-532.

ROMERO-ZARCO, C. -2013- Clasificación infragenérica del género Helictochloa Romero Zarco (Poaceae). Acta Bot. Malac. 38: 253-255.

ROMERO-ZARCO, C. -2014- Notas taxonómicas sobre el género Helictochloa Romero Zarco (Poaceae). Acta Bot. Malac. 39: 308-310.

RÖSER, M. -1989- Karyologische, systematische und chorologische Untersuchungen an der Gattum Helictotrichon Besser ex Schultes \& Schultes im westlichen Mittelmeergebiet. Diss. Bot. 145: 1-250.

RÖSER, M. -2006- Notes on the distribution and taxonomy of some S Iberian and Maghrebian perennial Aveneae [genera Helictotrichon Besser, Pseudarrhenatherum Rouy, Arrhenatherum P. Beauv. and Avenula (Dumort.) Dumort.]. Lagascalia 26: 140-147.

SAINT-YVES, A. -1931- Contribution à l'étude des Avena sect. Avenastrum (Eurasie et région méditeranéenne). Candollea 4: 353-504.

SENNEN, F. \& F. MAURICIO -1934- Catálogo florístico del Rif oriental y principalmente de las cábilas limitrofes con Melilla. Melilla.

Dirección del autor. Departamento de Biología Vegetal y Ecología, Facultad de Biología, Universidad de Sevilla. Apartado 1095, 41080-Sevilla (España). zarco@us.es 